\title{
Implications of Byproduct Chemistry in Nanoparticle Synthesis
}

Stappen, Frederick N.; Enemark-Rasmussen, Kasper; Junor, Glen P.; Clausen, Mads Hartvig; Zhang, Jingdong; Engelbrekt, Christian

Published in:

Journal of Physical Chemistry C

Link to article, DOI:

10.1021/acs.jpcc.9b03193

Publication date:

2019

Document Version

Peer reviewed version

Link back to DTU Orbit

Citation (APA):

Stappen, F. N., Enemark-Rasmussen, K., Junor, G. P., Clausen, M. H., Zhang, J., \& Engelbrekt, C. (2019). Implications of Byproduct Chemistry in Nanoparticle Synthesis. Journal of Physical Chemistry C, 123(41), 25402-25411. https://doi.org/10.1021/acs.jpcc.9b03193

\section{General rights}

Copyright and moral rights for the publications made accessible in the public portal are retained by the authors and/or other copyright owners and it is a condition of accessing publications that users recognise and abide by the legal requirements associated with these rights.

- Users may download and print one copy of any publication from the public portal for the purpose of private study or research.

- You may not further distribute the material or use it for any profit-making activity or commercial gain

- You may freely distribute the URL identifying the publication in the public portal

If you believe that this document breaches copyright please contact us providing details, and we will remove access to the work immediately and investigate your claim 


\section{Implications of Byproduct Chemistry in Nanoparticle}

\section{Synthesis}

Frederick N. Stappen ${ }^{\dagger}$, Kasper Enemark-Rasmussen ${ }^{\dagger}$, Glen P. Junor ${ }^{\ddagger}$, Mads H. Clausen ${ }^{\dagger,}$, Jingdong Zhang $^{\dagger}$, Christian Engelbrekt ${ }^{\dagger, 1 / *}$

†Technical University of Denmark, Department of Chemistry, Kemitorvet 207, 2800 Lyngby, Denmark.

$\ddagger$ Department of Chemistry and Biochemistry, University of California, San Diego, La Jolla, California 92093-0358, United States.

$\S$ Center for Nanomedicine and Theranostics, Kemitorvet 207, 2800 Lyngby, Denmark.

" Department of Chemistry, University of California Irvine, Irvine, California 92697-2025, United States.

ABSTRACT: Byproducts in metal nanoparticle synthesis can interfere with nanomaterial formation and self-assembly, as well as the perceived nanomaterial properties. Such syntheses go through a complicated series of intermediates making it difficult to predict byproduct chemistry, and challenging to determine experimentally. By a combined experimental and theoretical approach, the formation of organic byproducts are mapped out for the synthesis of gold nanoparticles with the Good's buffer MES. Comprehensive nuclear magnetic resonance studies supported by mass spectrometry, ultraviolet-visible spectroscopy, and density functional theory reveal a number of previously unidentified byproducts formed by oxidation, $\mathrm{C}-\mathrm{N}$ bond cleavage, and $\mathrm{C}-\mathrm{C}$ bond formation. A reaction mechanism involving up to four consecutive oxidations is proposed. Oligomeric products with 
electronic transitions in the visible range are suggested. This approach can be extended broadly, and lead to more informed synthesis design and materials characterization.

\section{INTRODUCTION}

Gold nanoparticles (AuNPs) are among the most studied nanomaterials and central to much research in nanotechnology and nanomedicine. ${ }^{1}$ Understanding the formation of both nanoparticles and organic byproducts is critical for rational design of the nanomaterials, particularly for healthcare and food industries. ${ }^{2-4}$ As an example, toxicity of nanomaterials has in some cases been linked to impurities deposited on the nanomaterial surface, which in turn influences the evaluation of the biocompatibility of nanomaterials, and make such studies very sensitive to purification methods. ${ }^{5}$ Furthermore, knowing the reaction mechanisms, and thus the organic products formed en route to the inorganic nanomaterials, will clarify the role of different components on behavior and properties of the systems. For instance, an organic byproduct was found to be responsible for the formation of superlattices during AuNP synthesis with $t$-butylamine,${ }^{6}$ while macroscale AuNP-coated fibers were formed from trimethylamine oxidation byproducts, ${ }^{7}$ highlighting the potential of byproducts to dictate the nanomaterial structure. Thus, understanding byproduct formation allows for more rational design and manipulation of nanomaterial syntheses. Finally, molecular-sized nanomaterials, such as nanoclusters, cannot easily be separated from organic byproducts, and are thus often applied without rigorous purification. $^{8-10}$ Since nanoparticle formation commonly occurs through non-selective redox chemistry, byproduct characterization is difficult, and often overlooked in nanomaterial synthesis, which may lead to incorrect conclusions based on the behavior of nanomaterial mixtures. ${ }^{11}$

Meanwhile, the drive for green syntheses of gold nanomaterials has led to the successful use of benign compounds as reducing and stabilizing agents, ${ }^{12-16}$ and resulted in a plethora of materials with wide-ranging properties. Generally, green synthesis of AuNPs is done by mixing tetrachloroaurate $\left[\mathrm{AuCl}_{4}\right]^{-}$with a reducing agent (e.g. $\mathrm{NaBH}_{4}{ }^{17}$ or glucose ${ }^{2}$ ) and a stabilizing agent (e.g. starch $^{2}$ ), or molecules acting as both, for example 2-( $N$-morpholino)ethanesulfonic acid (MES), ${ }^{11}$ citrate $^{18}$, tryptophan, ${ }^{14}$ or aniline. ${ }^{19,20}$ Green AuNPs are generally evaluated via their optical properties and morphology through ultraviolet-visible (UV-vis) spectroscopy and transmission electron microscopy 
(TEM), respectively. The properties are typically attributed to the gold nanostructures and sometimes the stabilizing agent. ${ }^{13-15}$ Several reports on the synthesis of gold nanoclusters (AuNCs) using heteroatom-rich small organic reducing agents present fluorescence as the main support for AuNC formation, ${ }^{8-10}$ while these transitions could be assigned to organic byproducts. ${ }^{11}$

One appealing reagent in AuNP synthesis is MES, as it serves multiple purposes simultaneously: reduction, buffering, and capping. Furthermore, it is frequently used in biochemistry as a non-toxic, and optically inactive buffer, and is generally considered inert. MES is used as buffer in biological AuNP-mediated $\mathrm{H}_{2} \mathrm{O}_{2}$ detection where $\mathrm{HAuCl}_{4}$ is reduced by $\mathrm{H}_{2} \mathrm{O}_{2}$, and formation of AuNPs detected by UV-vis spectroscopy. ${ }^{21-24}$ Even though it is often recognized that MES may itself reduce $\mathrm{HAuCl}_{4}$, the influence of the byproducts of this side-reaction on detection, or the biological systems themselves have not been considered. ${ }^{21-24}$

One possible reason for the dearth of mechanistic studies is the complex progression of gold intermediates, making it difficult to predict the fate of the organic reducing agent as well as its influence on the optical (and other) properties of the AuNP sols. ${ }^{2,25}$ Complicating the matter, all intermediates in gold nanostructure synthesis, including gold complexes ${ }^{26}$, nanoparticles ${ }^{27,28}$ and nanoclusters ${ }^{29}$, are known catalysts for reactions, such as the oxidation of amines to imines, ${ }^{30}$ oxidations with dioxygen, ${ }^{27}$ and CO oxidation. ${ }^{31}$

Despite the challenge, organic byproducts formed during AuNP synthesis are sometimes discussed, ${ }^{16}$ and can be supported by infrared (IR) absorption spectra, though the complexity of the resulting mixtures makes it difficult to reach firm conclusions. ${ }^{15,32}$ Newman and Blanchard studied AuNP formation with amine reducing agents by nuclear magnetic resonance (NMR) spectroscopy and identified polyaniline formation after reduction of $\mathrm{HAuCl}_{4}$ with aniline using ${ }^{1} \mathrm{H} \mathrm{NMR} .{ }^{20}$ Similarly, dense organic layers were observed around AuNPs when $\mathrm{HAuCl}_{4}$ was reduced by tryptophan, and poly-tryptophan from oxidative polymerization was suggested based on ${ }^{1} \mathrm{H}$ NMR and UV-vis spectroscopy. ${ }^{12,14}$ However, the sensitivity and resolution of standard ${ }^{1} \mathrm{H}$ NMR spectroscopy can be a limiting factor for looking at byproduct mixtures at low concentrations.

Regardless of the difficulties in characterizing organic byproducts in nanomaterial synthesis, such efforts are necessary because 1) byproducts may interfere with the perceived properties of the 
nanomaterials, 2) byproducts may associate strongly with the nanomaterials surfaces affecting their toxicity, and 3) byproducts can play an active role in the evolution of nanomaterial morphology.

The current work presents detailed characterization of the organic byproducts and chemistry involved in gold nanoparticle synthesis, as well as the effects of byproducts on the properties of nanoparticle sols. The MES-stabilized AuNPs studied here are conveniently removed by syringe filtration, confirmed by UV-vis spectroscopy and inductively coupled plasma optical emission spectroscopy (ICP-OES). ${ }^{11}$ We conduct a comprehensive NMR study supported by UV-vis spectroscopy, and mass spectrometry (MS) to examine the byproducts from $\mathrm{HAuCl}_{4}$ reduction by MES. A range of MES derivatives are identified, and a reaction mechanism with up to four consecutive oxidation steps is proposed. The electronic absorption spectra of identified intermediates and oligomers are simulated by time-dependent density functional theory (TD-DFT), and molecular dynamics (MD) simulations, supporting the formation of conjugated dimers and radicals.

\section{METHODS}

Synthesis of gold nanoparticle sols. AuNPs were synthesized in a $100 \mathrm{~mL}$ round-bottom flask under magnetic stirring in an oil bath by adding $20 \mathrm{mM} \mathrm{HAuCl}_{4}$ stock solution to MES in water at the target temperature (Figure 1a and Video S1). ${ }^{11,33}$ The concentrations of $\mathrm{HAuCl}_{4}$ and $\mathrm{MES}$ were varied. Three conditions were examined, i.e. standard ( $\left.2 \mathrm{mM} \mathrm{HAuCl}_{4}, 10 \mathrm{mM} \mathrm{MES}\right)$, high gold (10 mM $\mathrm{HAuCl}_{4}, 10 \mathrm{mM} \mathrm{MES}$ ), and high MES ( $\left.2 \mathrm{mM} \mathrm{HAuCl}_{4}, 75 \mathrm{mM} \mathrm{MES}\right)$ concentration. After a certain reaction time, the AuNPs were removed by filtration through 2 sequential $0.2 \mu$ polypropylene syringe filters (VWR international, Figure 1c and 1e) onto a petri dish or watch glass, where the sample was kept at $80{ }^{\circ} \mathrm{C}$ until dry. The dried powder was redissolved in $\mathrm{H}_{2} \mathrm{O}$ or $\mathrm{D}_{2} \mathrm{O}$ for $\mathrm{NMR}$ or chromatographic analysis. The efficiency of AuNP removal by the filtration method was verified by UV-vis spectra of the filtrates showing no detectable AuNP absorbance, and ICP-OES showing $>99.995 \%$ and $>99.974 \%$ removal of gold (corresponding to $\sim 0.08$ and $0.5 \mu \mathrm{M}$, Figure S4) for syntheses carried out at 21 and $80{ }^{\circ} \mathrm{C}$, respectively, in agreement with previous results. ${ }^{11}$ 


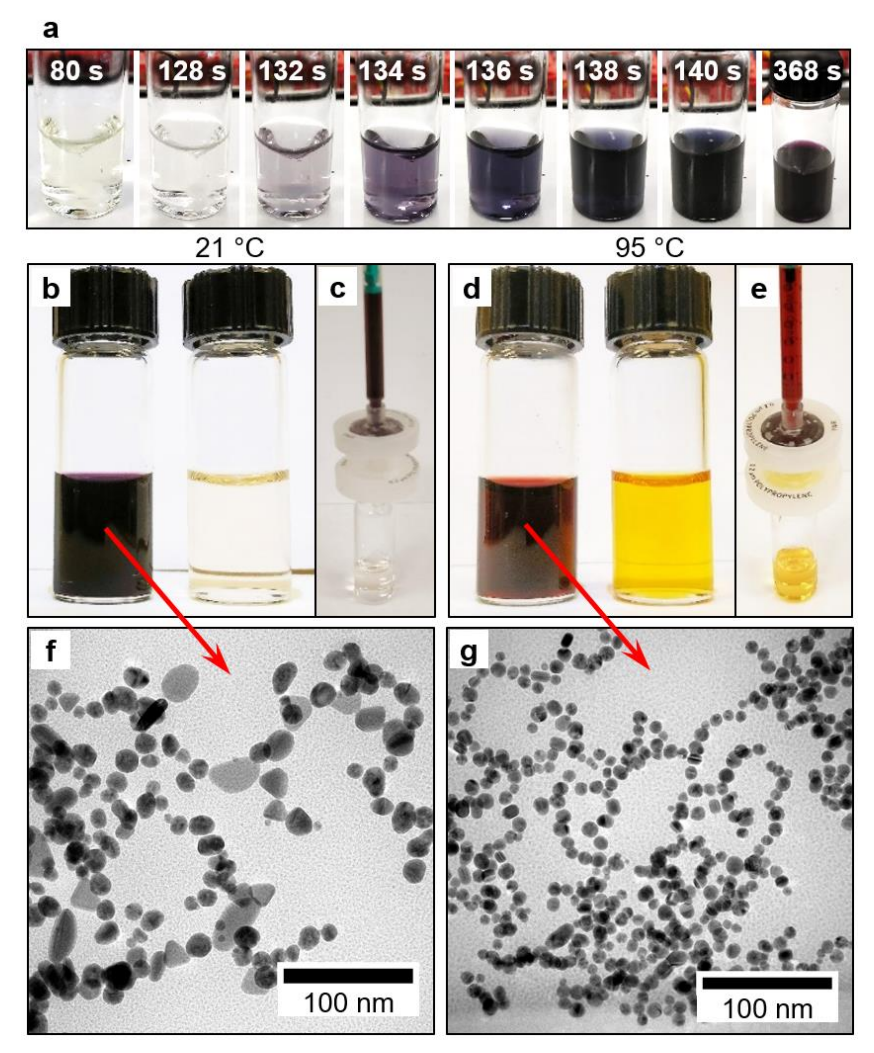

Figure 1. Synthesis, purification and nanostructure of AuNPs synthesized at (a-c, f) $21^{\circ} \mathrm{C}$, and (d-e, g) $95{ }^{\circ} \mathrm{C}$. (a) Photographs at different reaction times during the first 6 min of AuNP synthesis at 21 ${ }^{\circ} \mathrm{C}$. (b, d) Comparison of as-synthesized sol (left) and filtrate (right). (c, e) Syringe filtration showing the retention of AuNPs from the sol (in the syringe) by the filters. (f-g) TEM micrographs of AuNP product from synthesis at (f) $21^{\circ} \mathrm{C}$, and (g) $95^{\circ} \mathrm{C}$.

NMR spectroscopy. The presented NMR data were recorded on a Bruker AVANCEIII spectrometer operating at a ${ }^{1} \mathrm{H}$ frequency of $799.75 \mathrm{MHz}$ (Oxford Instruments superconducting magnet refitted for Bruker Biospin electronics). The system was equipped with a $5 \mathrm{~mm}$ TCI CryoProbe (Bruker Biospin) and all experiments were performed at $298 \mathrm{~K}$. Filtrates were re-dissolved in $600 \mu \mathrm{L} \mathrm{D}_{2} \mathrm{O}$, unless otherwise noted.

Ultraviolet-visible (UV-vis) spectroscopy. UV-vis spectra were recorded on an Agilent 8453 (Santa Clara CA, USA) or PerkinElmer Lambda 950 (Waltham MA, USA) spectrophotometer in quartz cuvettes. In situ UV-vis experiments were performed by mixing $\mathrm{HAuCl}_{4}$ and $\mathrm{MES}$ in a glass vial at room temperature (r.t.) with stirring for $4 \mathrm{~min}$ before transferring the solution to a $1 \mathrm{~mm}$ quartz cuvette.

Chromatography. Hydrophilic interaction liquid chromatography (HILIC) coupled to an electrospray (positive and negative, ES+ and ES-, respectively) single quadrupole mass spectrometer 
(MS), and photodiode array (PDA) detector was done on an Acquity UPLC system from Waters (Milford MA, USA).

Computational methods. The theoretical approach was conducted based on density functional theory (DFT). All geometry optimizations, frequency calculations, and time-dependent DFT simulations were performed with the Gaussian09 program suite. The best fitting parameters were found to be the $\omega B 97 X-D$ functional and def2-TZVPP basis set, benchmarked against several functionals and basis sets. Ground state geometries were optimized without constraints at the corresponding level of theory. Frequency calculations were performed to confirm the geometry was, in fact, a minimum (zero imaginary frequencies) at the corresponding level of theory. TD-DFT calculations ${ }^{34}$ with the TammDancoff approximation (TDA) ${ }^{35}$ were used to simulate UV-vis spectra of target compounds. MD simulations with a large number of explicit water molecules, in conjunction with TD-DFT, were used to demonstrate the importance of product-solvent interactions in accurate assignment of UV-vis transitions. Computational output files are available from the UCSD Library Digital Collections. ${ }^{36}$

Further experimental details are provided in the supplementary information.

\section{RESULTS AND DISCUSSION}

We studied organic byproducts from AuNPs prepared by adding $\mathrm{HAuCl}_{4}$ to $\mathrm{pH}$-adjusted $\mathrm{MES}$ solution at the target temperature. Facilitated by $\mathrm{N}-\mathrm{Au}$ complexation, $\left[\mathrm{AuCl}_{4}\right]^{-}$was rapidly reduced by MES, as observed with other amines,${ }^{20}$ as evidenced by the color change from the slight yellow color of the precursor to purple/red of the AuNPs (Figure 1a and Video S1). Figure 2 presents the dynamics of the optical properties of the AuNP-byproduct mixture. The peak at $520 \mathrm{~nm}$ in Figure $2 \mathrm{a}$ is characteristic of AuNPs, and arises from localized surface plasmon resonance (LSPR). Most noticeably, a prominent peak develops at $365 \mathrm{~nm}$, while the characteristic LSPR features of the AuNPs remain unchanged over five hours. It is thus clear that two distinct phases occur during the synthesis: one is fast and dominated by AuNP-forming reactions, and the second is a slower phase during which the AuNP size/shape does not change. Removing the AuNPs from the mixture by filtration at different reaction times (Figure 2b) shows the evolution of several distinct absorption bands, and an isosbestic point at $316 \mathrm{~nm}$. Removing the contribution from AuNPs to the spectra in Figure 2a (by subtraction 
of the initial spectrum, $4 \mathrm{~min}$ ) reveals additional details of the dynamics, and bands that change independently at 200, 235, 300,360, and $475 \mathrm{~nm}$, Figure 2c and 2d. We note that Figure 2c agrees well with Figure $2 \mathrm{~b}$ supporting the validity of this treatment. When the AuNPs are physically removed by filtration immediately after formation ( 3 min, Figure 1 and Figure $\mathrm{S} 1$ ), the byproduct absorbance dynamics and composition are changed, Figure $2 \mathrm{e}$ and $2 \mathrm{f}$. The difference in background below 300 $\mathrm{nm}$ is caused by the species (mainly MES) present at $4 \mathrm{~min}$, which are removed by subtraction in Figure 1c. It is clear that the AuNPs affect the product distribution, and are involved in the formation of the species absorbing at $360 \mathrm{~nm}$ indicating a catalytic role of the AuNPs. Furthermore, it indicates that the byproducts associate with the AuNP surface since certain byproducts only form when AuNPs are not removed after their formation. Indications of byproduct-AuNP association during the fast AuNP-forming phase is also found in the TEM images, where non-spherical AuNPs (rods/plates) are found when synthesized at $21{ }^{\circ} \mathrm{C}$ (Figure 1f), as reported earlier. ${ }^{2} \mathrm{UV}$-vis spectra of reaction filtrates from a variety of synthesis conditions ( $\mathrm{pH}$, reactant concentrations, temperature) were investigated showing a strong dependence of relative and total concentrations of byproducts, Figure S6. The origin of these absorption peaks has not previously been reported, and we have previously observed how AuNP sols derived from reduction of $\left[\mathrm{AuCl}_{4}\right]^{-}$with MES exhibit special optical ${ }^{11}$ properties that cannot be explained simply by AuNPs or MES-derived $N$-oxide, as reported for MES oxidation by hydrogen peroxide. ${ }^{37}$ The current work aims at understanding the reaction, and role of organic byproducts. 

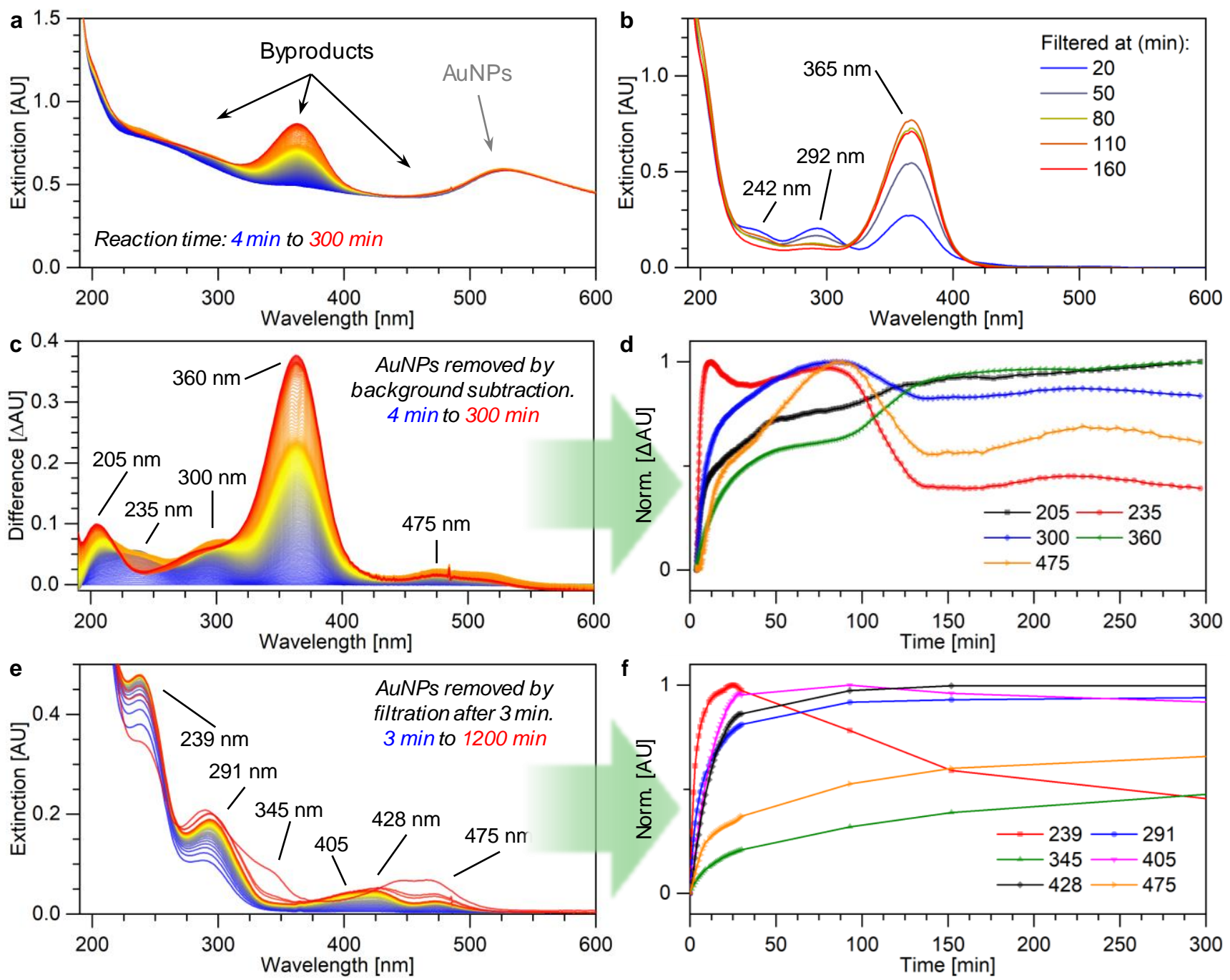

Figure 2. Observation of byproduct dynamics following AuNP formation at $21{ }^{\circ} \mathrm{C}$. (a) Evolution of the extinction spectrum of the AuNP sol from 4 to 300 min. (b) Absorbance of AuNP-free filtrates prepared after 20 to 160 min of reaction. (c) Difference spectra generated by subtracting the initial spectrum, 4 min, in (a) from the following spectra revealing a series of absorptions bands. (d) Timedependent intensity of peaks labelled in (c). (e) Evolution of the absorption spectrum of the filtrate when AuNPs are physically removed by filtration after 3 min. (f) Time-dependent intensity of peaks labelled in (e).

\section{Chemistry of MES oxidation in AuNP synthesis}

Our experimental results (discussed in the following section) suggest that the reaction involves up to four consecutive oxidation steps of MES. Scheme 1 presents our proposed reaction mechanism, where intermediates confirmed by NMR, MS, and X-ray crystallography are highlighted in blue. Unobserved intermediates in the reaction scheme are believed to be short-lived species necessary for 
the ultimate formation of oxalic acid. The reaction is initiated by coordination of $\left[\mathrm{AuCl}_{4}\right]^{-}$, or the aqua/hydroxy species such as $\left[\mathrm{AuCl}_{3} \mathrm{OH}_{2}\right]$ and $\left[\mathrm{AuCl}_{3} \mathrm{OH}\right]^{-38,39}$ to the nitrogen in MES. Reaction can only be observed at initial $\mathrm{pH}$ above 6 where the nitrogen in MES is deprotonated $(\mathrm{pKa}=6.15)$, and has a free lone pair.

Scheme 1. Proposed reaction mechanism of gold-catalyzed oxidation of MES to $N-(2-$ sulfoethyl)ethanolamine and oxalic acid. ${ }^{\mathrm{a}}$

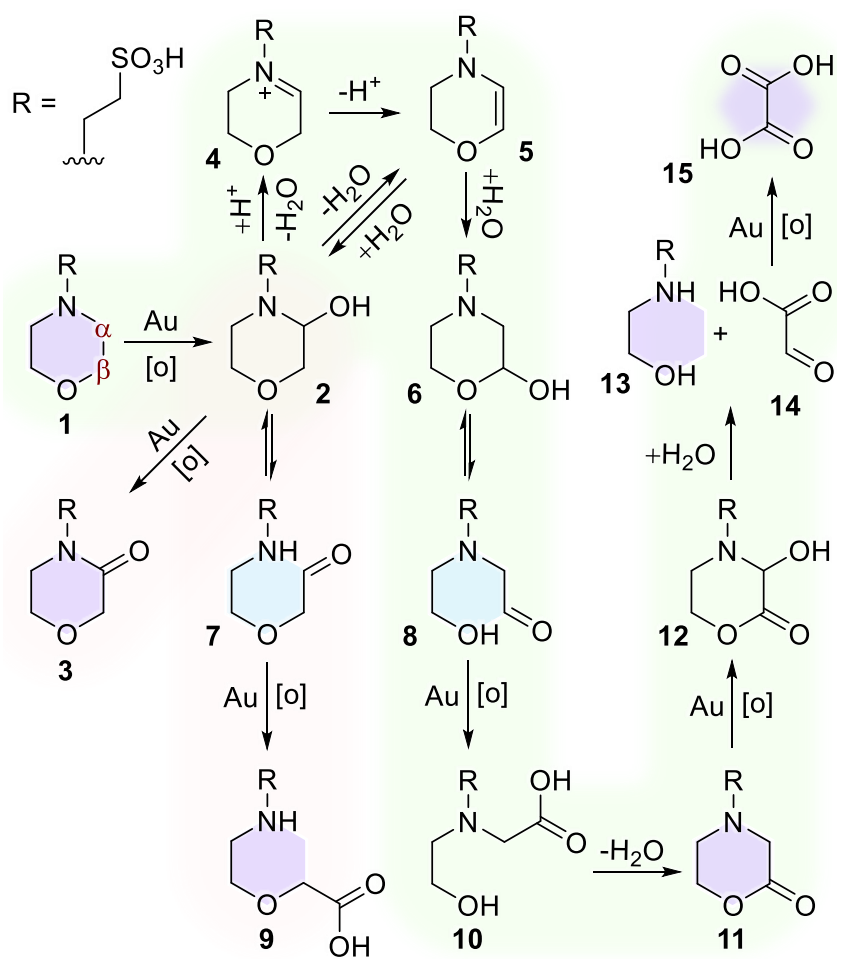

${ }^{a}$ The structure of purple compounds has been determined by NMR/HILIC-MS. Blue indicates support from in situ NMR experiments. Oxalic acid has been observed in separate experiments by Xray diffraction, Figure S23.

In the product mixtures, carbon atoms $\alpha$ and $\beta$ to the nitrogen are oxidized, and carbon-heteroatom bonds are broken. The first step is the oxidation of MES, either directly to hemiaminal (2) as shown or through an intermediate $\mathrm{N}$-oxide as reported previously. ${ }^{40}$ The iminium ion (4) is formed by dehydration, and loss of a proton forms the dihydro-oxazine (5). Hydration of the enamine leads to two possible isomeric aldehydes $(7,8)$ that are presumed to be easily oxidized to their corresponding acids $(9,10)$ under the reaction conditions. Cyclization and further oxidation eventually leads to the $N$-substituted ethanolamine (13) and free oxalic acid (15). Furthermore, the intermediates presented in Scheme 1 may undergo additional oxidation adding to the byproduct distribution. The oxidation 
steps presented in Scheme 1 can be driven by both $\mathrm{Au}(\mathrm{III}) / \mathrm{Au}(\mathrm{I})$, and dioxygen as oxidizing agents. AuNPs are known to catalyze oxidations with dioxygen as oxidant also after the gold precursor is consumed. In fact, Della Pina et al. demonstrated efficient conversion of tertiary amines to $N$-oxides under conditions similar to those in our system $\left(2 \mathrm{~atm} \mathrm{O}_{2},<90{ }^{\circ} \mathrm{C}\right.$, water $) .{ }^{41}$ Conversion and selectivity was affected by presence of alkali metals. A recent report from the Mizuno-Yamaguchi Lab showed selective $\alpha$-oxidation of tertiary amines with 1 bar $\mathrm{O}_{2}$ as terminal oxidant $\left(80{ }^{\circ} \mathrm{C}\right.$, water). ${ }^{42}$ They propose a similar reaction pathway to Scheme 1, where a lactam is formed via an intermediate iminium ion and a hemiaminal, and they also observed aldehyde/acid byproducts. It was suggested that $\beta-\mathrm{H}$ elimination of a coordinated amine/hemiaminal generates $\mathrm{Au}-\mathrm{H}$, the oxidation of which may produce oxygen-radicals. These reactions are likely to occur in our MES-AuNP sols, and therefore contribute to the byproduct formation.

\section{Identification of byproducts by NMR and HILIC-MS/PDA}

Structure identification of organic molecules with NMR spectroscopy generally employs a range of homonuclear and heteronuclear $2 \mathrm{D}$ experiments in addition to the standard $1 \mathrm{D}{ }^{1} \mathrm{H}$ and ${ }^{13} \mathrm{C}$ experiments. Here, we studied the organic byproducts from AuNP synthesis with a combination of 2D experiments to identify unique products and elucidate their structure. Hetero single quantum correlation (HSQC) shows the direct connection between protons and carbon atoms, whereas hetero multiple bond correlation (HMBC) provides long-range connections (generally between $2-4$ bonds). Homonuclear correlation spectroscopy (COSY), and total correlation spectroscopy (TOCSY) almost explicitly identifies 3-bond connections of the type $\mathrm{H}-\mathrm{C}-\mathrm{C}-\mathrm{H}$.

The structural similarities of byproducts complicates the analysis due to chemical shift overlap and signal crowding in the spectra. However, an advantage is that the compounds exhibit similar geometries and thereby equal-sized j-couplings. Thus, it will generally always be possible to distinguish $\mathrm{O}-\mathrm{CH}_{2}, \mathrm{~N}-\mathrm{CH}_{2}$ and $\mathrm{S}-\mathrm{CH}_{2}$ based on the observed 2D correlations. Furthermore, a comparison with the ${ }^{1} \mathrm{~J}_{1 \mathrm{H} 13 \mathrm{C}}$ couplings determined for the parent MES molecule serve as confirmations of the heteroatom- $\mathrm{CH}_{2}$ identification in addition to chemical shifts. 


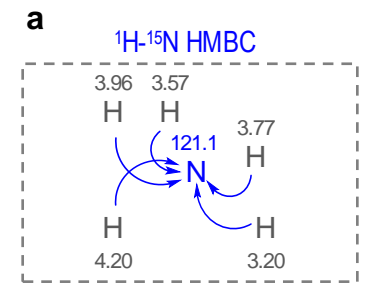

1) Protons coupling to nitrogen

b
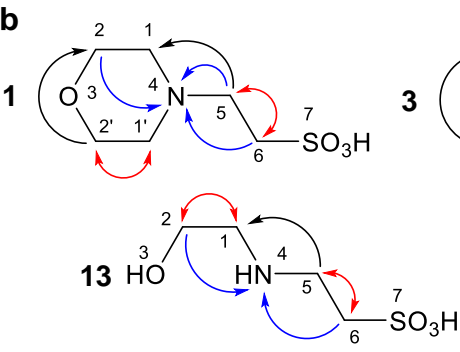

${ }^{1} \mathrm{H}-{ }^{15} \mathrm{~N} H M B C{ }^{1} \mathrm{H}-{ }^{13} \mathrm{C}$ HMBC HSQC-TOCSY/COSY

3

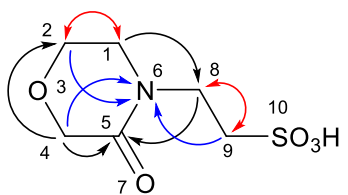

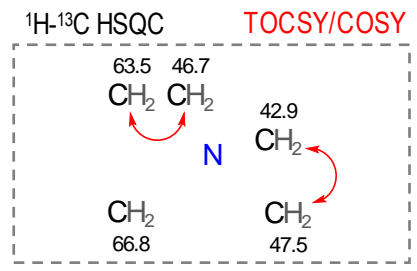

2) Protons attached 3) $\mathrm{CH}_{2}$ groups to carbons

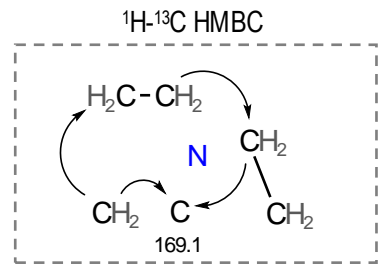

4) Fragments connected across heteroatoms

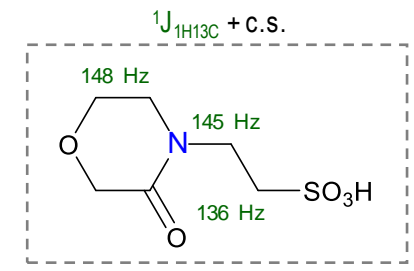

5) Funct. groups identified
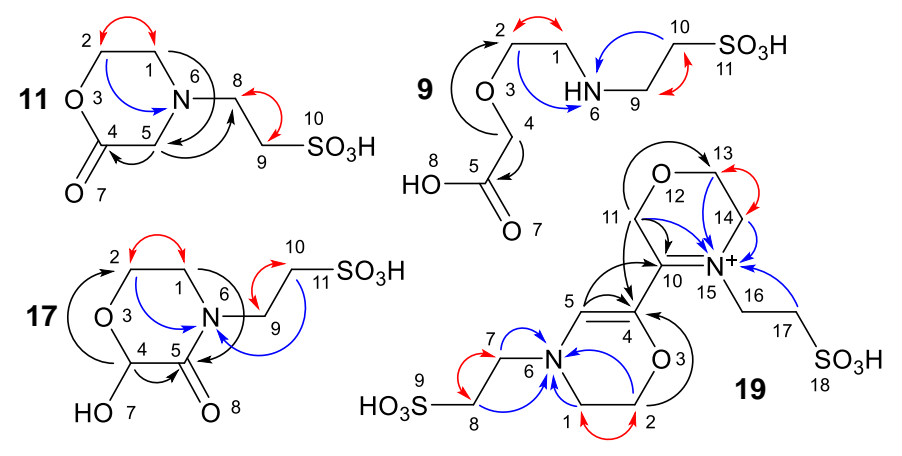

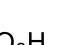

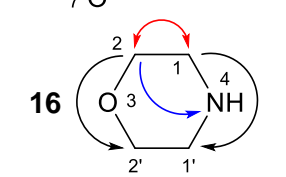
(n)

Figure 3. (a) Procedure followed for byproduct structure elucidation by NMR. The data employed in a step is indicated above it. c.s. = chemical shift. (b) Molecular structures of organic byproducts identified with NMR. The most important correlations observed with $2 \mathrm{D}$ NMR, i.e. ${ }^{1} \mathrm{H}-{ }^{15} \mathrm{~N}$ HMBC (blue), HSQC-TOCSY or -COSY (red), and ${ }^{1} \mathrm{H}-{ }^{13} \mathrm{C}$ HMBC (black), are indicated with arrows. All chemical shifts are reported in SI, Table S1-Table S11

Elucidation of the structures of compounds in the filtrate mixtures follows an almost identical procedure as illustrated in Figure 3a. As an example, the procedure for compound $\mathbf{3}$ is described, vide infra. A nitrogen atom is observed with a ${ }^{15} \mathrm{~N}$ chemical shift of $121.1 \mathrm{ppm}$ in the ${ }^{1} \mathrm{H}_{-}{ }^{15} \mathrm{~N}$ HMBC spectrum with five ${ }^{1} \mathrm{H}$ correlations at ${ }^{1} \mathrm{H}$ chemical shifts of $4.20,3.96,3.77,3.57$ and $3.20 \mathrm{ppm}$ (blue arrows in Figure 3) as seen in Figure 4f. These protons are then matched with ${ }^{13} \mathrm{C}$ chemical shifts of 66.8, 63.5, 42.9, 46.7 and $47.5 \mathrm{ppm}$, respectively, in the edited ${ }^{1} \mathrm{H}-{ }^{13} \mathrm{C}$ HSQC spectrum (Figure 4a), in-which the phases of the correlation peaks further show that all of the hydrogens are in fact $\mathrm{CH}_{2}$ groups. From the ${ }^{1} \mathrm{H}_{-}{ }^{13} \mathrm{C}$ HSQC-TOCSY and ${ }^{1} \mathrm{H}-{ }^{1} \mathrm{H}$ COSY experiments shown in Figure $4 \mathrm{~g}$ and $4 \mathrm{~h}$, correlations are observed at $\left[\delta_{1 \mathrm{H}} ; \delta_{1 \mathrm{H}}\right]=[3.96 ; 3.57]$ and $[3.77 ; 3.20] \mathrm{ppm}($ red arrows in Figure 3$)$, while the $\mathrm{H}$ with $\delta_{1 \mathrm{H}}=4.20 \mathrm{ppm}$ does not show any correlations. Key HMBC correlations to link together the fragments are observed at $\left[\delta_{1 \mathrm{H}} ; \delta_{13 \mathrm{C}}\right]=[4.20 ; 63.5],[4.20 ; 169.1],[3.77 ; 169.1]$ and [3.57; 42.9] ppm (Figure 4c and S11). Considering the ${ }^{1} \mathrm{H},{ }^{13} \mathrm{C}$ and ${ }^{15} \mathrm{~N}$ chemical shifts as well as HMBC correlations the lactam structure with the illustrated labeling then fits with the NMR 
observations. In addition, the ${ }^{1} \mathrm{~J}_{1 \mathrm{H} 13 \mathrm{C}}$ are found to be $136 \mathrm{~Hz}$ for position $9,145 \mathrm{~Hz}$ for position 8 and $148 \mathrm{~Hz}$ for position 2, which agree with the values determined for similar $\mathrm{CH}_{2}$-groups in MES.
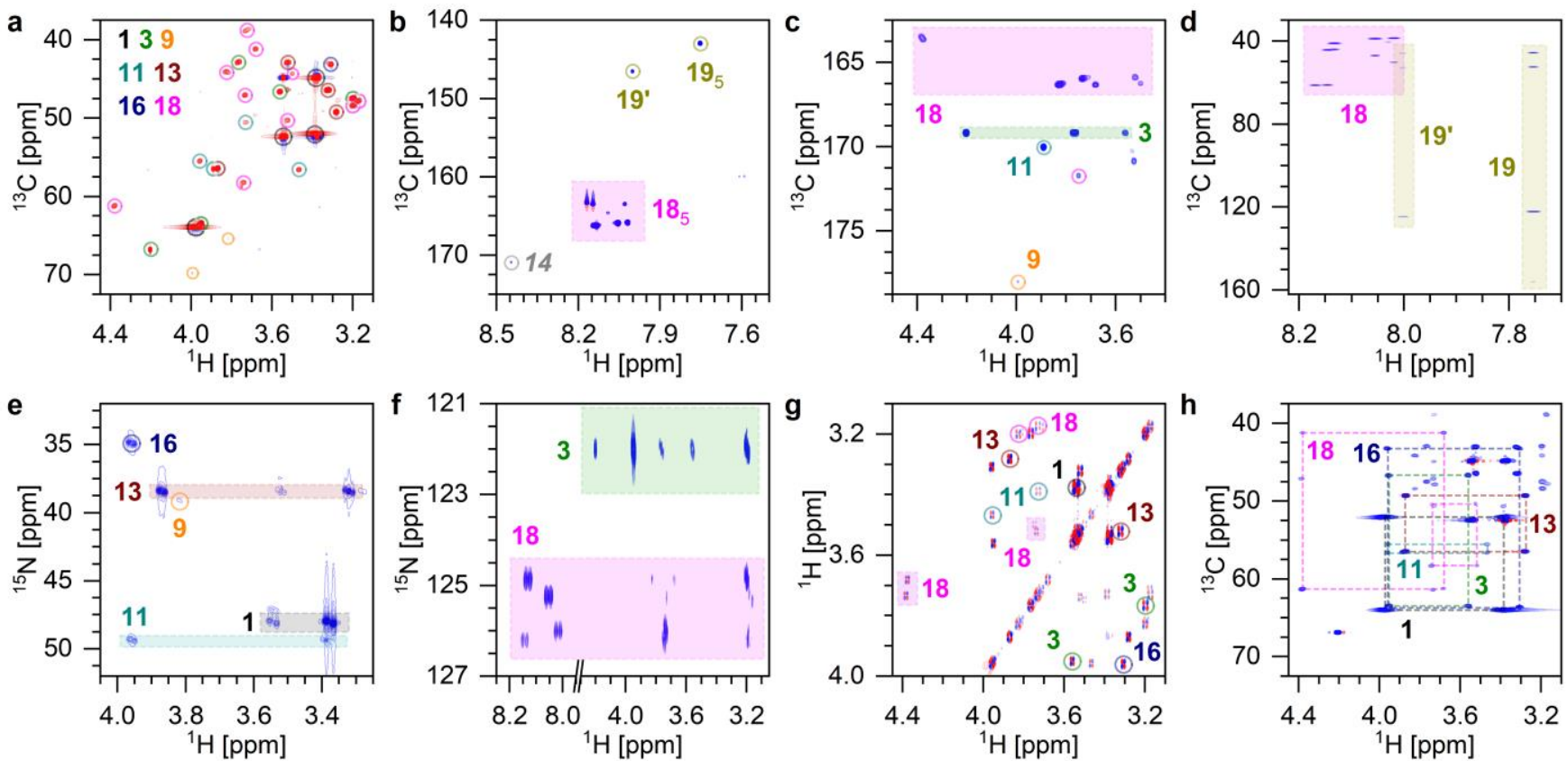

Figure 4. 2D NMR data. Bold numbers refer to compound numbers, and associated peaks are indicated with a color matching the compound number. ${ }^{1} \mathrm{H}-\left\{{ }^{13} \mathrm{C}\right\}$ HSQC data in the (a) low, and (b) high chemical shift range, ${ }^{1} \mathrm{H}-\left\{{ }^{13} \mathrm{C}\right\}$ HMBC data in the (c) low, and (d) high proton chemical shift range, ${ }^{1} \mathrm{H}-\left\{{ }^{15} \mathrm{~N}\right\}$ HMBC data in the (e) low, and (f) high nitrogen chemical shift range, $(\mathrm{g}){ }^{1} \mathrm{H}-\left\{{ }^{1} \mathrm{H}\right\}$ COSY data, and (h) ${ }^{1} \mathrm{H}-\left\{{ }^{13} \mathrm{C}\right\}$ TOCSY data. Full-range plots without annotations are given in Figure S9-S13.

Although this approach is straight-forward for abundant compounds, the heteronuclear experiments suffer from low signal intensity for compounds present in minute amounts, and at the same time stronger signals can mask weaker signals in the homonuclear experiments due to the reduced chemical shift range of ${ }^{1} \mathrm{H}$ compared to ${ }^{13} \mathrm{C}$ and ${ }^{15} \mathrm{~N}$. Thus, we only expect a complete structural assignment for the most abundant compounds, which should correspond to long-lived intermediates in the proposed reaction mechanism. Signals from intermediate products might still be observed with some NMR experiments, but missing ${ }^{1} \mathrm{H}-{ }^{13} \mathrm{C}$ and ${ }^{1} \mathrm{H}-{ }^{15} \mathrm{~N}$ HMBC correlations will result in structural ambiguities. The ${ }^{1} \mathrm{H}$ spectra of the AuNP filtrates are very convoluted, and the primary compound is unreacted MES 1. The bulk of the ${ }^{1} \mathrm{H}$ signal intensity is located from 2.8 to $4.2 \mathrm{ppm}$, and minor peaks are observed between 5 and 9 ppm, Figure S7. The narrow ${ }^{1} \mathrm{H}$ NMR chemical shift range limits the use of 2D homonuclear correlation experiments (i.e. COSY, TOCSY and nuclear Overhauser effect 
spectroscopy, NOESY). Instead, we rely primarily on 2D heteronuclear experiments where the wide chemical shift range of ${ }^{13} \mathrm{C}$ and ${ }^{15} \mathrm{~N}$ allow for most correlation peaks to be resolved. However, the low natural abundance of ${ }^{13} \mathrm{C}$ and ${ }^{15} \mathrm{~N}$ makes these experiments inherently less sensitive than the homonuclear experiments. Particularly the ${ }^{1} \mathrm{H}_{-}{ }^{15} \mathrm{~N}$ HMBC experiment detects only the major components of the mixtures, but the high resolution in the ${ }^{15} \mathrm{~N}$ dimension offers the most reliable way of assembling C-C fragments identified from the HSQC-TOCSY and ${ }^{1} \mathrm{H}_{-}{ }^{13} \mathrm{C}$ HMBC spectra.

Nine prominent ${ }^{15} \mathrm{~N}$ chemical shifts are observed in Figure 4e and 4f (Table S1, and Figure S13) with eight exhibiting correlation peaks to multiple ${ }^{1} \mathrm{H}$ resonances. Four of these fit with the assignments for compounds 3, 9, 11 and $\mathbf{1 3}$ with key correlations indicated in Figure $3 \mathrm{~b}$, and the observed ${ }^{15} \mathrm{~N}$ chemical shifts agree well with what is expected for amines and lactams. ${ }^{43}$ Furthermore, the expected $\mathrm{m} / \mathrm{z}$ values for these four byproducts $(210,228$ and 170 for positive ions of $\mathbf{3 / 1 1}, \mathbf{9} / \mathbf{1 0}$ and 13, respectively) are identified with HILIC-MS/PDA, Table S3-Table S6 and Figure S18-S19. A ${ }^{15} \mathrm{~N}$ shift at $35.6 \mathrm{ppm}$, and associated heteronuclear correlations were assigned to free morpholine 16. This was confirmed by spiking the sample, and indicates breaking of the $\mathrm{N}-\mathrm{C}$ bond to the ethylsulfonate, which may occur similarly to the formation of 7 (Scheme 1). The spectral overlap in the ${ }^{1} \mathrm{H}$ spectrum makes peak integration unreliable. However, the amount of unreacted MES as well as compound 3, 9, 11 and $\mathbf{1 3}$ can be estimated from the peak areas in the $1 \mathrm{D}{ }^{13} \mathrm{C}$ spectrum (acquired with inverse-gated ${ }^{1} \mathrm{H}$ decoupling), where peak overlap is less pronounced. The carbon signals from MES account for around $75 \%$ of the total signal intensity, while peaks assigned to $\mathbf{3 ,} \mathbf{9}, \mathbf{1 1}$ and $\mathbf{1 3}$ contribute with ca. 5, 3, 3 and 7\%, respectively. Considering the number of oxidation steps for these molecules, a total of $\sim 9 \mathrm{mM}$ electrons have been donated, which is significantly more than what can be accepted by the gold precursor $(6 \mathrm{mM})$. This corroborates that MES undergoes several oxidation steps, possibly with dioxygen as oxidant in addition to the gold precursor.

Several ${ }^{1} \mathrm{H}$ methine signals with varying intensities are observed with ${ }^{1} \mathrm{H}$ NMR in a chemical shift range between 5 and $7 \mathrm{ppm}$. For most of these signals the intensity is too low to observe long-range heteronuclear correlations excluding a full structural assignment. Only for the most intense signal at a ${ }^{1} \mathrm{H}$ chemical shift of $5.24 \mathrm{ppm}$ it is possible to reliably assign the corresponding hydrogen atom to position 4 in compound $\mathbf{1 7}$. For signals between 5 and $6 \mathrm{ppm}$, all ${ }^{1} \mathrm{H}-{ }^{13} \mathrm{C}$ HSQC correlations fall in a 
${ }^{13} \mathrm{C}$ chemical shift range of 95 and 115 ppm (Figure S9). These chemical shifts suggest the presence of a hydroxyl group on either the $\alpha$ or $\beta$ position in the morpholine ring as in compounds 2 and $\mathbf{6}$. Further, it is possible to identify two distinct TOCSY correlations for several of the ${ }^{1} \mathrm{H}$ signals in this region, consistent with an equilibrium between morpholinols with an axial and an equatorial hydroxyl substituent, stemming from mutarotation. These are most likely all secondary alcohols originating from 2 or 6 with additional modifications on the neighboring carbon atom. The higher shifted peaks between 7 and 8 ppm exhibit ${ }^{13} \mathrm{C}$-HSQC correlations in a chemical shift range of 120 to 140 indicating the presence of a double bond as in $\mathbf{5}$.

Six ${ }^{1} \mathrm{H}$ signals belonging to distinct coupling systems are observed around a chemical shift of $8 \mathrm{ppm}$ with ${ }^{13} \mathrm{C}$-HSQC correlations between 163 and 166 ppm, Figure 4b Table S9. Four of these coupling systems exhibit strong ${ }^{15} \mathrm{~N}$ 2-bond $\mathrm{HMBC}$ correlations to highly-shifted nitrogen atoms with ${ }^{15} \mathrm{~N}$ chemical shifts between 124.8 and $126.2 \mathrm{ppm}$, Figure 4f. Based on the observed heteronuclear and homonuclear NMR correlations, we propose that the signals originate from two isomeric dimers formed from enamines with an $\alpha-\beta$ double-bond. A symmetric (O-O) dimer gives rise to two coupling systems (18a and $\mathbf{1 8 b})$ due to the presence of two conformers. The four remaining coupling systems $(\mathbf{1 8 c}-\mathbf{1 8 f})$ arise from an asymmetrical $(\mathrm{N}-\mathrm{O})$ dimer, presumably also existing as two conformers in equilibrium. The structural assignment of the dimers are supported by HILIC-MS (Table S10), where ions corresponding to didehydro-MES-dimers have been observed in all filtrates from syntheses carried out at $21^{\circ} \mathrm{C}$.

In addition to the six ${ }^{1} \mathrm{H}$ signals arising from dimer-type byproducts discussed above, a seventh $1 \mathrm{H}$ signal at $7.76 \mathrm{ppm}$ is present in fresh samples, and not found after a few days. From data obtained at early stages of the byproduct formation, the structure could be solved as an asymmetric dimer with conjugation connecting the two identified nitrogen atoms at $\delta_{15 \mathrm{~N}}=119$ and $133 \mathrm{ppm}$ (compound 19 Figure 3 and Table S11). A set of HMBC correlations similar to compound $\mathbf{1 9}$ are found. Further correlations in the HSQC and NOESY spectra were found for the strongest sets, 19' and 19', while full elucidation was not possible (Figure 4b, Figure S15, Table S12). 
Several weak ${ }^{1} \mathrm{H}$ signals were observed at 9-10 ppm, which is usually associated with aldehyde hydrogens. Low intensity prevented resolution of 2D correlations to these signals. Further details of the byproduct formation kinetics were sought through an in situ NMR experiment. The reactants were mixed directly in the NMR tube immediately prior to the initiation of the in situ experiment, and ${ }^{1} \mathrm{H}$ spectra were recorded every $90 \mathrm{~s}$ over $20 \mathrm{~h}$ with a starting delay of 3 minutes for locking and shimming of the instrument. Here, a ${ }^{1} \mathrm{H}$ (triplet) signal at $9.56 \mathrm{ppm}$ is observed in the first few spectra but decay to noise-level within the first 5 min (Figure S15). This supports the presence of aldehydes, such as 7 and $\mathbf{8}$, that are rapidly oxidized to carboxylic acids $(\mathbf{9} / \mathbf{1 0})$. The peak area of the enamine proton in 19 increased exponentially with a time constant of 80 min (Figure S15). The intensity started dropping over a few days. A similar decrease in intensity was observed for the asymmetrical (N-O) dimers (18c18f) ${ }^{1} \mathrm{H}$ signals, while the two ${ }^{1} \mathrm{H}$ signals attributed to the symmetric $(\mathrm{O}-\mathrm{O})$ dimers both increased in intensity with time.

Further support for dimer-type species was found with diffusion ordered spectroscopy (DOSY) experiments, which allows for the association of diffusion coefficients to 1D NMR signals. Straightforward analysis was challenged by large concentration differences, weak signals, signal crowding at a ${ }^{1} \mathrm{H}$ shift range (3-4 ppm) but ${ }^{1} \mathrm{H}$ signals of 1, 16, 18 and 19 could be isolated (Figure S16). We found that diffusion coefficients for the signals we associate with dimers (18 and 19) are smaller than those of compounds similar to MES, while the smallest coefficient was found for $\mathbf{1 6}$ morpholine, in good agreement with expectations. A signal is observed at a ${ }^{1} \mathrm{H}$ chemical shift of $8.4 \mathrm{ppm}$ with an HSQC correlation to a ${ }^{13} \mathrm{C}$ chemical shift of $170.9 \mathrm{ppm}$ (Figure $4 \mathrm{~b}$ ), and no additional $2 \mathrm{D}$ correlations. In the DOSY experiment, this ${ }^{1} \mathrm{H}$ signal is associated with a diffusion coefficient slightly larger than that of morpholine 16. We therefore speculate that this signal could originate from the proposed compound 14 (glyoxalic acid) or a similar derivative.

\section{Simulation of optical properties of AuNP synthesis byproducts}

After identifying the major byproducts of the reaction, we sought to understand their impact on the UV-vis spectrum. TD-DFT was employed to simulate absorption spectra of the identified intermediates, as well as additional target molecules identified by HILIC-MS/PDA (Table S13). First, 
spectra of species presented in Scheme 1 were simulated together with compounds previously proposed, ${ }^{11}$ Figure S24. Only four molecules exhibited transitions above $200 \mathrm{~nm}$, i.e. two iminium ions, the dihydro-oxazine and the bicyclic sultone, Figure 5a.
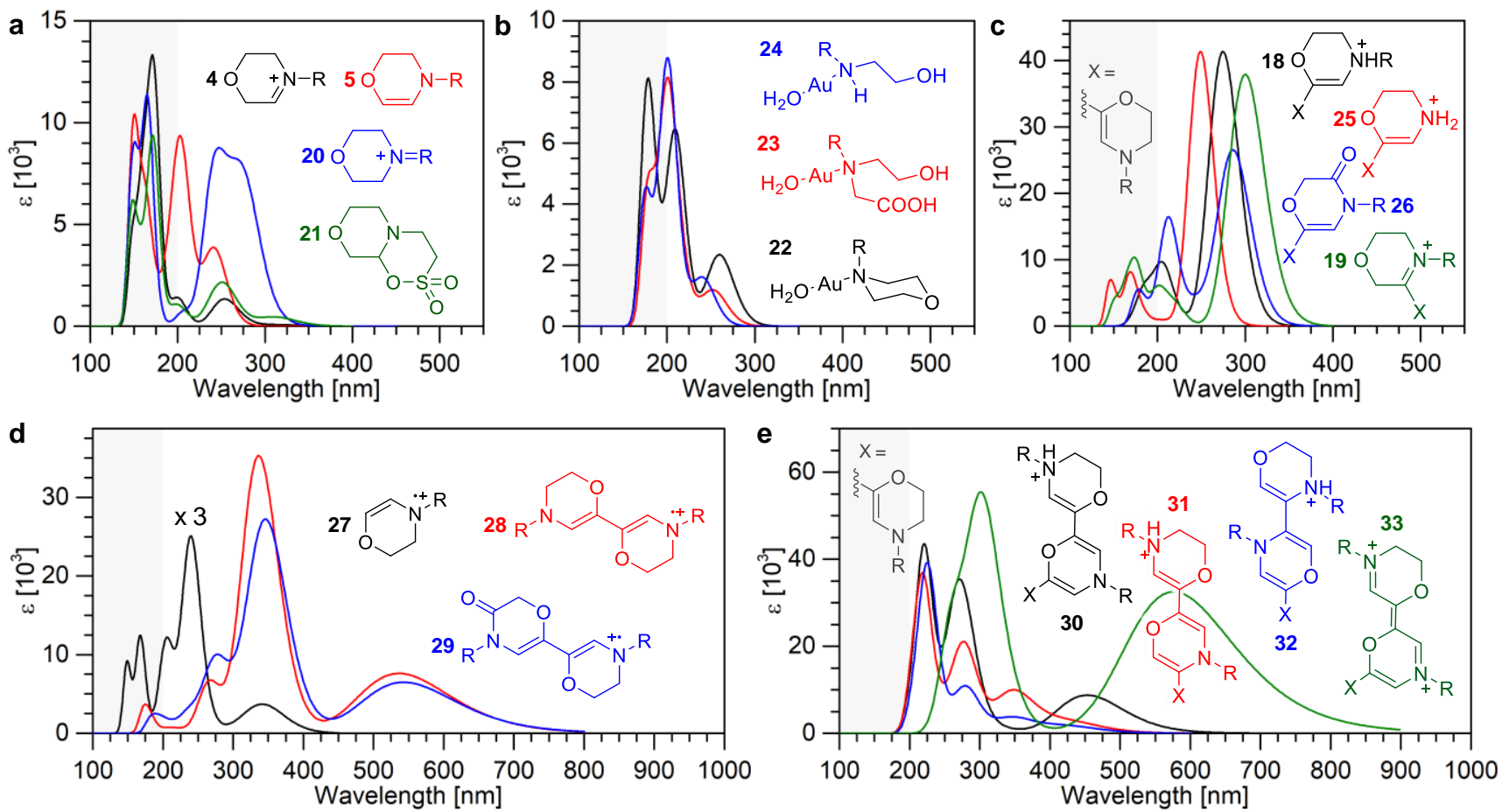

Figure 5. TD-DFT calculated absorption spectra of selected MES oxidation byproducts with transitions in the UV range. (a) reduced molecular weight species, (b) Au(I) complexes, (c) dimertype species, (d) radical cations, and (e) trimer-type species. The grey area lies outside the range of the UV-vis spectrometer. $R$ represents ethylsulfonate, and $X$ in (c) and (e) represents compound 5 bonded through the $\beta$-carbon.

During structural optimization of $\mathbf{2 1}$ the sultone bond was broken forming the iminium ion $\mathbf{4}$ where the sulfonate chain was folded back towards the charged nitrogen. All compounds in Figure 5a are expected to be detected with the same $\mathrm{m} / \mathrm{z}$ in $\mathrm{ES}+(\mathrm{m} / \mathrm{z}=194)$, which is indeed observed with HILICMS, Figure S21. A weak UV peak around 250-260 nm is found in HILIC-PDA at the same elution time similar to what is predicted with TD-DFT.

Trace amounts of gold $(<1 \mu \mathrm{M})$ was found in the filtrates. ${ }^{11}$ Though these levels are not expected to be sufficient to explain the UV-vis data, absorption spectra of $\mathrm{Au}(\mathrm{I})$ complexes with the identified amines were simulated, Figure S25. Only aquaaurum(I) complexes exhibited transitions in the UV range, Figure $5 \mathrm{~b}$. The molar extinction coefficients are small as expected for $5 \mathrm{~d}^{10} \mathrm{Au}(\mathrm{I})$ systems, 
confirming that the absorption bands do not originate from $\mathrm{Au}(\mathrm{I})$ complexes. To account for transitions in organic molecules down to $2.5 \mathrm{eV}(500 \mathrm{~nm})$, it is necessary to extend delocalization significantly indicating the formation of larger products through coupling of MES-derived species. NMR provided indications of products possibly formed from $\mathbf{5}$ coupled via either of the double-bonded carbons, Table S9.

Several dimer targets were identified from HILIC-MS, and their spectra calculated by TD-DFT. All protonation states were evaluated for species with several protonatable nitrogens. The full sets are provided in Figure S26 while the species expected to be most abundant in the filtrates are presented in Figure 5c. The simple dimer 18 correlates with m/z 385 found in HILIC-MS where the PDA spectrum is dominated by a peak at $360 \mathrm{~nm}$, Figure S20.The calculated spectrum of 18 shows a sharp peak at $275 \mathrm{~nm}$, and no transitions around the observed peak at $360 \mathrm{~nm}$. A compound with m/z 277 and UV absorption at $260 \mathrm{~nm}$ has been observed in HILIC-MS consistent with the didehydro-MES/didehydromorpholine dimer 25, and in excellent agreement with the calculated spectrum of $\mathbf{2 6}$. The dimer identified in the in situ NMR experiment, 19, showed a single peak in our spectrometer window centered at $300 \mathrm{~nm}$.

Radical cation formation by chemical or electrochemical oxidation of Good's buffers was identified by Grady et al. using electron paramagnetic resonance spectroscopy (EPR). ${ }^{44}$ Later, MES-derived radicals were reported by Habib et al. using $\left[\mathrm{AuCl}_{4}\right]^{-}$as the oxidant indicating a different reaction mechanism with the gold precursor from that of earlier oxidants. ${ }^{45,46}$ Recently, the Odom group used EPR to follow the formation of radicals during AuNP formation with Good's buffers as reducing agents. ${ }^{47}$ They were able to link the radical formation to the development of anisotropic AuNP morphology. The exact structure of the MES-derived radicals has not been reported yet. This led us to look at possible stable radical cations in our system, and calculate the spectra of radical cation forms of monomer 5, and dimers 18 and 26, i.e. the radicals 27, 28 and 29 (Figure 5d). As expected, the radicals display a significant redshift of the lowest energy transitions relative to the non-radicals. Structure 28 gives an intense peak at $335 \mathrm{~nm}$ as well as a broad peak around $530 \mathrm{~nm}$. The spectrum of 29 was very similar to $\mathbf{2 8}$ but slightly redshifted with the main intensity at $345 \mathrm{~nm}$. This coincides with the peak observed in solutions of MES-derived radicals, which was attributed to the formation of nitro- 
compounds. ${ }^{45}$ No sign of nitro-compounds were found in our NMR data. Thus, our data suggest that this absorbance arises from dimer-type radicals such as $\mathbf{2 8}$ and 29. Oxidation can occur on both sides of the morpholine leading to potential higher oligomers. Several trimers were evaluated showing that cross-conjugated (30), and fully conjugated (33) species support visible transitions, Figure 3e and Figure S26, as observed after $80{ }^{\circ} \mathrm{C}$ reaction, Figure S6. Polymeric byproducts have also been observed for AuNP synthesis with other amine reducing agents, e.g tryptophan, ${ }^{12,14}$ triethylamine, ${ }^{7}$ and 2-methylaniline ${ }^{48}$.

The suggested and identified molecules are very hydrophilic consisting of several polar and charged functional groups. Water molecules are therefore expected to interact closely with the byproducts in aqueous solution. It is known that DFT has difficulty modelling solvation effects in many systems. ${ }^{49}$

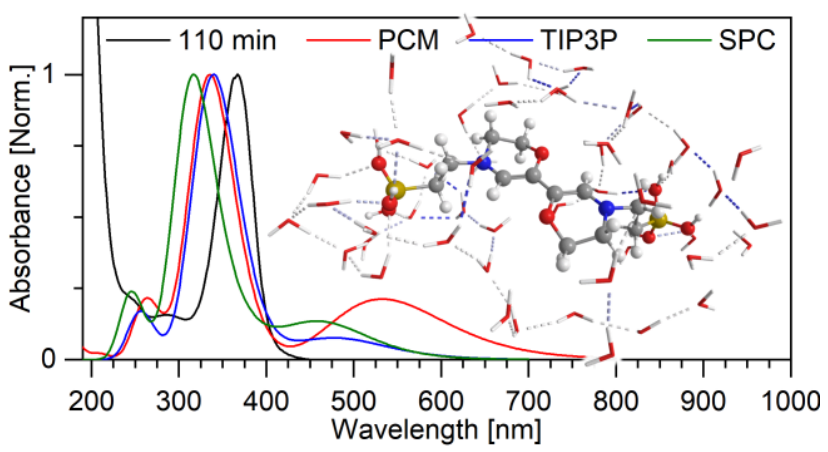

Figure 6. Comparison of experimental spectrum of the filtrate at $110 \mathrm{~min}$ (black) to TD-DFT simulated spectra of $\mathbf{2 8}$ with PCM solvent model (red), with 50 water molecules after 250 frames of the MD run with the TIP3P water model (blue), and 500 frames of the MD run with SPC (green). The inset shows 28 surrounded by 50 water molecules after 500 frames using SPC.

Therefore, we attempted molecular dynamics (MD) simulations to explicitly account for solvent interactions using our best candidate for the UV peak at $360 \mathrm{~nm}$, compound $\mathbf{2 8}$. The system included 1133 water molecules surrounding the rigid, optimized structure of $\mathbf{2 8}$, in a truncated octahedron with $10 \AA$ radius (more details in SI). The spectra of $\mathbf{2 8}$ with the 50 nearest water molecules from several frames of the MD experiment were simulated with TD-DFT, Figure S31. This rigorous approach to solvation leads to a decrease in intensity and blue-shift of the lowest energy peak, Figure 6, resulting in a good match to experimental spectra (which we note are highly convoluted as seen in Figure 2 and Figure S6, making accurate assessment of the peak positions difficult). We therefore propose that 
dimer radical cations are responsible for the main UV-vis absorbance of the filtrates (as observed with UV-vis spectroscopy and HILIC-PDA), and that these convert to dimers such as $\mathbf{1 8}$ in the electrospray which is then detected with HILIC-MS. A radical may initially be formed by reduction of Au(I) during AuNP formation, which subsequently reacts with MES-derived molecules to form $\mathbf{2 8}$ at a longer timescale than that of AuNP formation. Radicals can also form via Au-H species as proposed by Jin et al. ${ }^{42}$

In general, oxidized MES monomers and dimers exhibit UV absorption bands below $300 \mathrm{~nm}$, while radicals and trimers absorb up to $600 \mathrm{~nm}$. Such convoluted UV-vis spectra have previously been reported for solutions obtained by reducing $\mathrm{HAuCl}_{4}$ with $\mathrm{MES}$ at high concentrations $(\approx 50$ and 170 $\mathrm{mM}$, respectively). ${ }^{50}$ Absorption bands at 290, 330, 366 and $475 \mathrm{~nm}$ were observed in good agreement with our results. These peaks were assigned to several types of AuNCs but no other data were presented to support AuNCs. In later studies, the same solutions were used to investigate the biocompatibility of AuNCs. ${ }^{51}$ The MES-derived solutions showed high cytotoxicity in contrast to other non-toxic AuNCs, and increased reactive oxygen species (ROS) generation. We suspect that the reported optical properties (and possibly cytotoxicity) arise from MES-derived byproducts such as radicals and oligomers, rather than AuNCs, further stressing the importance of understanding organic byproducts in nanomaterials synthesis. In fact, DNA cleavage caused by Good's buffer-derived radicals has been reported. ${ }^{46}$ These radicals formed by reaction with $\mathrm{Au}(\mathrm{III})$ but not with other metal ions.

\section{CONCLUSION}

It is important to consider non-colloid byproducts in solution-based syntheses of nanomaterials especially in the field of green nanomaterials, where mild, organic reducing agents are oxidized by reactive metal precursors. The chemistry in these highly dynamic environments is much more complicated than what is typically assumed. Considering organic byproducts can strengthen the understanding of nanomaterial properties, evaluation of their toxicity, and prediction of nanomaterial morphology. We have presented an in-depth analysis of byproduct mixtures from the synthesis of AuNPs with the common Good's buffer MES as a reducing agent. AuNP-free filtrates were 
investigated extensively by a range of 2D NMR techniques, in situ UV-vis spectroscopy, and HILICMS/PDA. The structures of a number of highly oxidized byproducts have been determined, and a reaction mechanism proposed. A single MES molecule undergoes up to four consecutive 2-electron oxidations to ultimately form 2-((2-hydroxyethyl)amino) ethane-1-sulfonic acid and oxalic acid. UVvis spectroscopy shows that a series of compounds with electronic transitions between 200 and 515 $\mathrm{nm}$ form during and after AuNP formation, and NMR data indicated formation of dimeric byproducts with extended conjugation. Target compounds were identified based on HILIC-MS data, our proposed mechanism and previous studies on Good's buffer oxidation. Their absorption spectra were calculated with TD-DFT, and evaluated against UV-vis and HILIC-MS/PDA data. We believe that oligomerization of didehydro-MES occurs leading to dimers and trimers with transitions in the range 250-600 nm. Furthermore, these compounds can form radical cations that are stable at $21^{\circ} \mathrm{C}$ for weeks. Specifically, the dimer radical 28 gives rise to strong UV absorbance at $360 \mathrm{~nm}$. This compound appears after the formation of AuNPs, and is thus not formed directly through reaction with the gold precursor. The approach presented here is generally applicable to other nanomaterial syntheses, and demonstrates how comprehensive NMR, MS and DFT can be combined to describe very complicated byproduct mixtures. Such efforts are important for accurate evaluation of nanomaterial properties, and crucial for the application of nanomaterials, e.g. in nanotechnology, nanomedicine and food industries.

\section{ASSOCIATED CONTENT}

Supporting information. Supplementary experimental details and results, video of AuNP synthesis. This material is available free of charge via the Internet at http://pubs.acs.org.

\section{AUTHOR INFORMATION}

\section{Corresponding Author}

* cheng@kemi.dtu.dk 


\section{ACKNOWLEDGEMENTS}

Financial support from the Independent Research Fund Denmark to JZ (DFF-1335-00330 and DFF 4093-00297), CE (DFF-5054-00107) and MHC (DFF-7017-00026B), the Lundbeck Foundation to JZ (R141-2013-13273), and the Alfred P. Sloan Foundation University Center for Exemplary Mentoring to GPJ is greatly appreciated. The $800 \mathrm{MHz}$ and $600 \mathrm{MHz}$ NMR data were recorded at the NMR Center at DTU supported by the Villum Foundation. We acknowledge the computational resources made available by the W. M. Keck Foundation. This material is based upon work supported by the National Science Foundation Graduate Research Fellowship Program under Grant No. (DGE1650112; GPJ). Any opinions, findings, and conclusions or recommendations expressed in this material are those of the author(s) and do not necessarily reflect the views of the National Science Foundation. We sincerely thank Dr. Samuel Gilbert Eliot, and Assoc. Prof. Charlotte Held Gotfredsen at the Department of Chemistry, DTU for valuable discussions of the NMR data, Prof. Francesco Paesani at the Department of Chemistry and Biochemistry, UCSD for discussion and guidance on the computational work, Eleftherios Lambros at the Department of Chemistry and Biochemistry, UCSD for guidance on MD simulations, and Assoc. Prof. Kenny Ståhl at the Department of Chemistry, DTU for acquisition and analysis of single-crystal X-ray diffraction. Electron microscopy work was performed at the UC Irvine Materials Research Institute (IMRI).

\section{REFERENCES}

1. Yang, X.; Yang, M.; Pang, B.; Vara, M.; Xia, Y. Gold Nanomaterials at Work in Biomedicine. Chem. Rev. 2015, 115, 10410-10488.

2. Engelbrekt, C.; Jensen, P. S.; Sørensen, K. H.; Ulstrup, J.; Zhang, J. Complexity of Gold Nanoparticle Formation Disclosed by Dynamics Study. J. Phys. Chem. C 2013, 117, 7-9.

3. Smolkova, B.; El Yamani, N.; Collins, A. R.; Gutleb, A. C.; Dusinska, M. Nanoparticles in Food: Epigenetic Changes Induced by Nanomaterials and Possible Impact on Health. Food Chem. Toxicol. 2015, 77, 64-73. 
4. Bouwmeester, H.; Dekkers, S.; Noordam, M. Y.; Hagens, W. I.; Bulder, A. S.; de Heer, C.; ten Voorde, S. E. C. G.; Wijnhoven, S. W. P.; Marvin, H. J. P.; Sips, A. J. A. M. Review of Health Safety Aspects of Nanotechnologies in Food Production. Regul. Toxicol. Pharmacol. 2009, 53, $52-62$.

5. Harper, B.; Sinche, F.; Ho Wu, R.; Gowrishankar, M.; Marquart, G.; Mackiewicz, M.; Harper, S. The Impact of Surface Ligands and Synthesis Method on the Toxicity of Glutathione-Coated Gold Nanoparticles. Nanomaterials 2014, 4, 355-371.

6. Wu, S.; Ang, C. Y.; Luo, Z.; Yu Tan, S.; Nguyen, K. T.; Zhao, Y. Byproduct-Induced in-Situ Formation of Gold Colloidal Superparticles. Sci China Mater 2015, 58, 860-866.

7. Kuo, P. L.; Chen, C. C. Generation of Gold Thread from Au(III) and Triethylamine. Langmuir 2006, 22, 7902-7906.

8. Bao, Y.; Yeh, H. C.; Zhong, C.; Ivanov, S. A.; Sharma, J. K.; Neidig, M. L.; Vu, D. M.; Shreve, A. P.; Dyer, R. B.; Werner, J. H.; et al. Formation and Stabilization of Fluorescent Gold Nanoclusters Using Small Molecules. J. Phys. Chem. C 2010, 114, 15879-15882.

9. Jiang, J.; Gao, P.; Zhang, Y.; Zhang, G.; Zhou, Y.; Dong, C.; Shuang, S. Rapid One-Pot Synthesis of MMTA Protected Fluorescent Gold Nanoclusters for Selective and Sensitive Detection of Ferric Ion. Talanta 2017, 174, 44-51.

10. Zheng, S.; Yin, H.; Li, Y.; Bi, F.; Gan, F. One-step Synthesis of L-Tryptophan-Stabilized Dual-Emission Fluorescent Gold Nanoclusters and Its Application for Fe3+sensing. Sensors Actuators, B Chem. 2017, 242, 469-475.

11. Engelbrekt, C.; Wagner, M.; Christiansen, M. U.-B.; Christensen, H. E. M.; Qian, X.; Ulstrup, J.; Zhao, C.; Zhang, J. Side Effect of Good's Buffers on Optical Properties of Gold Nanoparticle Solutions. ChemElectroChem 2016, 3, 1212-1218.

12. Selvakannan, P. R.; Mandal, S.; Phadtare, S.; Gole, A.; Pasricha, R.; Adyanthaya, S. D.; Sastry, M. Water-Dispersible Tryptophan-Protected Gold Nanoparticles Prepared by the Spontaneous 
Reduction of Aqueous Chloroaurate Ions by the Amino Acid. J. Colloid Interface Sci. 2004, $269,97-102$.

13. Kasture, M.; Sastry, M.; Prasad, B. L. V. Halide Ion Controlled Shape Dependent Gold Nanoparticle Synthesis with Tryptophan as Reducing Agent: Enhanced Fluorescent Properties and White Light Emission. Chem. Phys. Lett. 2010, 484, 271-275.

14. Iosin, M.; Baldeck, P.; Astilean, S. Study of Tryptophan Assisted Synthesis of Gold Nanoparticles by Combining UV-Vis, Fluorescence, and SERS Spectroscopy. J. Nanoparticle Res. 2010, 12, 2843-2849.

15. Rawat, K. A.; Kailasa, S. K. Sensors and Actuators B: Chemical 4-Amino Nicotinic Acid Mediated Synthesis of Gold Nanoparticles for Visual Detection of Arginine, Histidine, Methionine and Tryptophan. Sensors Actuators B. Chem. 2016, 222, 780-789.

16. Zou, L.; Qi, W.; Huang, R.; Su, R.; Wang, M.; He, Z. Green Synthesis of a Gold NanoparticleNanocluster Composite Nanostructures Using Trypsin as Linking and Reducing Agents. $A C S$ Sustain. Chem. Eng. 2013, 1, 1398-1404.

17. Zhao, P.; Li, N.; Astruc, D. State of the Art in Gold Nanoparticle Synthesis. Coord. Chem. Rev. 2013, 257, 638-665.

18. Turkevich, J.; Stevenson, P. C.; Hillier, J.; Turkevich, J.; Stevenson, P. C. A Study of the Nucleation and Growth Processes I N the Synthesis Of. Discuss. Faraday Soc. 1951, 11, 55.

19. Mallick, K.; Witcomb, M. J.; Scurrell, M. S. Polyaniline Stabilized Highly Dispersed Gold Nanoparticle: An in-Situ Chemical Synthesis Route. J. Mater. Sci. 2006, 41, 6189-6192.

20. Newman, J. D. S.; Blanchard, G. J. Formation of Gold Nanoparticles Using Amine Reducing Agents. Langmuir 2006, 22, 5882-5887. 
21. de la Rica, R.; Bat, E.; Herpoldt, K. L.; Xie, H. N.; Bertazzo, S.; Maynard, H. D.; Stevens, M. M. Nanoparticle Growth via Concentration Gradients Generated by Enzyme Nanopatterns. Adv. Funct. Mater. 2014, 24, 3692-3698.

22. de la Rica, R.; Stevens, M. M. Plasmonic ELISA for the Ultrasensitive Detection of Disease Biomarkers with the Naked Eye. Nat. Nanotechnol. 2012, 8, 1759-1764.

23. Gobbo, P.; Biondi, M. J.; Feld, J. J.; Workentin, M. S. Arresting the Time-Dependent H2O2 Mediated Synthesis of Gold Nanoparticles for Analytical Detection and Preparative Chemistry. J. Mater. Chem. B 2013, 1, 4048.

24. Wu, W. B.; Zhan, L.; Wang, J.; Huang, C. Z. A Plasmon Resonance Light Scattering Assay of Glucose Based on the Formation of Gold Nanoparticles. Anal. Methods 2014, 6, 3779.

25. Laramy, C. R.; Fong, L. K.; Jones, M. R.; O’Brien, M. N.; Schatz, G. C.; Mirkin, C. A. Understanding Nanoparticle-Mediated Nucleation Pathways of Anisotropic Nanoparticles. Chem. Phys. Lett. 2017, 683, 389-392.

26. Homogeneous Gold Catalysis; Slaughter, L. M., Ed.; Springer International Publishing: Heidelberg New York Dordrecht London, 2015.

27. Turner, M.; Golovko, V. B.; Vaughan, O. P. H.; Abdulkin, P.; Berenguer-Murcia, A.; Tikhov, M. S.; Johnson, B. F. G.; Lambert, R. M. Selective Oxidation with Dioxygen by Gold Nanoparticle Catalysts Derived from 55-Atom Clusters. Nature 2008, 454, 981-983.

28. Della Pina, C.; Falletta, E. Gold-Catalyzed Oxidation in Organic Synthesis: A Promise Kept. Catal. Sci. Technol. 2011, 1, 1564.

29. Jin, R.; Zeng, C.; Zhou, M.; Chen, Y. Atomically Precise Colloidal Metal Nanoclusters and Nanoparticles: Fundamentals and Opportunities. Chem. Rev. 2016, 116, 10346-10413.

30. Zhu, B.; Lazar, M.; Trewyn, B. G.; Angelici, R. J. Aerobic Oxidation of Amines to Imines Catalyzed by Bulk Gold Powder and by Alumina-Supported Gold. J. Catal. 2008, 260, 1-6. 
31. Peng, S.; Lee, Y.; Wang, C.; Yin, H.; Dai, S.; Sun, S. A Facile Synthesis of Monodisperse Au Nanoparticles and Their Catalysis of CO Oxidation. Nano Res. 2008, 1, 229-234.

32. Annadhasan, M.; Kasthuri, J.; Rajendiran, N. Green Synthesis of Gold Nanoparticles under Sunlight Irradiation and Their Colorimetric Detection of Ni 2+ and Co 2+ Ions. RSC Adv. 2015, 5, 11458-11468.

33. Engelbrekt, C.; Sørensen, K. H.; Zhang, J.; Welinder, A. C.; Jensen, P. S.; Ulstrup, J. Green Synthesis of Gold Nanoparticles with Starch-glucose and Application in Bioelectrochemistry. J. Mater. Chem. 2009, 19, 7839.

34. Adamo, C.; Jacquemin, D. The Calculations of Excited-State Properties with Time-Dependent Density Functional Theory. Chem. Soc. Rev. 2013, 42, 845-856.

35. Chantzis, A.; Laurent, A. D.; Adamo, C.; Jacquemin, D. Is the Tamm-Dancoff Approximation Reliable for the Calculation of Absorption and Fluorescence Band Shapes? J. Chem. Theory Comput. 2013, 9, 4517-4525.

36. Stappen, F.; Enemark-Rasmussen, K.; Junor, G. P.; Clausen, M. H.; Zhang, J.; Engelbrekt, C. DOI: 10.6075/J0GB228D. UCSD Library Digital Collections; 2018.

37. Zhao, G.; Chasteen, N. D. Oxidation of Good's Buffers by Hydrogen Peroxide. Anal. Biochem. 2006, 349, 262-267.

38. Fry, F. H.; Hamilton, G. A.; Turkevich, J. The Kinetics and Mechanism of Hydrolysis of Tetrachloroaurate(III). Inorg. Chem. 1966, 5, 1943-1946.

39. Peck, J. A.; Tait, C. D.; Swanson, B. I.; Brown, G. E. Speciation of Aqueous Gold(Iii) Chlorides from Ultraviolet Visible Absorption and Raman Resonance Raman Spectroscopies. Geochim. Cosmochim. Acta 1991, 55, 671-676.

40. Zhao, G.; Chasteen, N. D. Oxidation of Good's Buffers by Hydrogen Peroxide. Anal. Biochem. 2006, 349, 262-267. 
41. Pina, C. Della; Falletta, E.; Rossi, M.; Della Pina, C.; Falletta, E.; Rossi, M. Selective Oxidation of Tertiary Amines on Gold Catalysts. Top. Catal. 2007, 44, 325-329.

42. Jin, X.; Kataoka, K.; Yatabe, T.; Yamaguchi, K.; Mizuno, N. Supported Gold Nanoparticles for Efficient $\alpha$-Oxygenation of Secondary and Tertiary Amines into Amides. Angew. Chem., Int. Ed. 2016, 55, 7212-7217.

43. Webb, G. A. In Encyclopedia of Spectroscopy and Spectrometry; Lindon, J. C., Ed.; Elsevier Ltd.: Oxford, UK, 2010; pp 1790-1799.

44. Grady, J. K.; Chasteen, N. D.; Harris, D. C. Radicals from "Good's” Buffers. Anal. Biochem. 1988, $173,111-115$.

45. Habib, A.; Tabata, M.; Wu, Y. G. Formation of Gold Nanoparticles by Good's Buffers. Bull. Chem. Soc. Jpn. 2005, 78, 262-269.

46. Tabata, M.; Habib, A.; Watanabe, K. DNA Cleavage by Good's Buffers in the Presence of Au(III). Bull. Chem. Soc. Jpn. 2005, 78, 1263-1269.

47. Chandra, K.; Rugg, B. K.; Ratner, M. A.; Wasielewski, M. R.; Odom, T. W. Detecting and Visualizing Reaction Intermediates of Anisotropic Nanoparticle Growth. J. Am. Chem. Soc. 2018, 140, 3219-3222.

48. Subramaniam, C.; Tom, R. T.; Pradeep, T. On the Formation of Protected Gold Nanoparticles from AuCl4- by the Reduction Using Aromatic Amines. J. Nanoparticle Res. 2005, 7, 209217.

49. Riera, M.; Brown, S. E.; Paesani, F. Isomeric Equilibria, Nuclear Quantum Effects, and Vibrational Spectra of $\mathrm{M}+(\mathrm{H} 2 \mathrm{O}) \mathrm{n}=1-3$ Clusters, with $\mathrm{M}=\mathrm{Li}, \mathrm{Na}, \mathrm{K}, \mathrm{Rb}$, and $\mathrm{Cs}$, through Many-Body Representations. J. Phys. Chem. A 2018, 122, 5811-5821.

50. Matulionyte, M.; Marcinonyte, R.; Rotomskis, R. Photoinduced Spectral Changes of Photoluminescent Gold Nanoclusters. J. Biomed. Opt. 2014, 20, 051018. 
51. Matulionyte, M.; Dapkute, D.; Budenaite, L.; Jarockyte, G.; Rotomskis, R. Photoluminescent Gold Nanoclusters in Cancer Cells: Cellular Uptake, Toxicity, and Generation of Reactive Oxygen Species. Int. J. Mol. Sci. 2017, 18, 1-17. 


\section{TOC graphic}

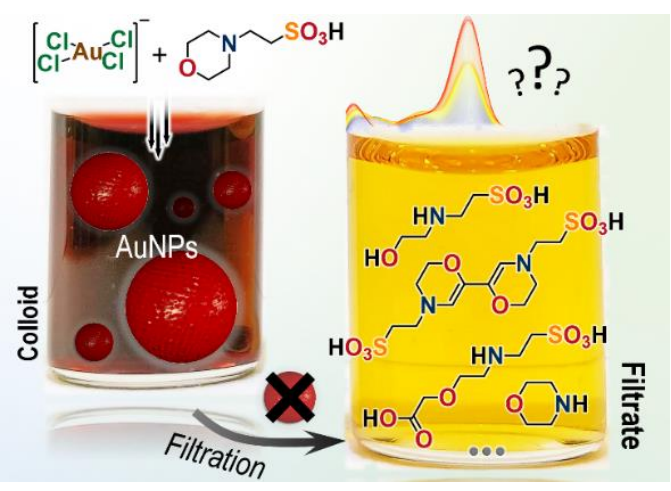

